\title{
Autonomic Nervous System Assessment in Critically Ill Patients Undergoing a Cognitive Rehabilitation Therapy
}

\author{
David Hernando 1,2 , Marc Turon ${ }^{3,4}$, Raquel Bailón ${ }^{1,2}$, Sol Fernandez-Gonzalo ${ }^{3}$, Jesús Lázaro ${ }^{1,2}$, \\ Gemma Gomà ${ }^{3,5}$, Eduardo Giil ${ }^{1,2}$, Jaume Montanyà ${ }^{4}$, Josefina López-Aguilar ${ }^{3,4,5}$, Candelaria de \\ Haro $^{4,5}$, Pablo Laguna ${ }^{1,2}$, Lluís Blanch ${ }^{3,4,5}$ \\ ${ }^{1}$ Biomedical Signal Interpretation \& Computational Simulation (BSICoS) Group, Aragón Institute of \\ Engineering Research (I3A), IIS Aragón, University of Zaragoza, Zaragoza, Spain \\ ${ }^{2}$ CIBER de Bioingeniería, Biomateriales y Nanomedicina (CIBER-BBN), Zaragoza, Spain \\ ${ }^{3}$ Research Department, Institut d'Investigació i Innovació Parc Taulí (I3PT), Fundació Parc Taulí, \\ Corporació Sanitària Parc Taulí, Univ. Institute, Univ. Autònoma de Barcelona, Sabadell, Spain \\ ${ }^{4}$ CIBER Enfermedades Respiratorias (CIBERES), Instituto de Salud Carlos III, Madrid, Spain \\ ${ }^{5}$ Critical Care Center, Hospital de Sabadell, Corporació Sanitària Universitaria Parc Taulí, Universitat \\ Autònoma de Barcelona, Sabadell, Spain
}

\begin{abstract}
Recent clinical and electrophysiological studies reveal a high incidence of autonomic nervous system (ANS) dysfunction in patients treated in Intensive Care Units (ICUs). Cognitive rehabilitation $(C R)$ is a behavioral therapy that has proven to be effective improving cognitive deficits in clinical populations with abnormalities in brain activation patterns. A total of 17 critically ill patients received $C R$ aimed to improve the ANS status, which was quantified in terms of HRV. The CR included cognitive exercises aimed to improve prefrontal activation. HRV was obtained during pre-CR, CR and post-CR. Power in the low (PLF) and high $(P H F)$ frequency bands related to sympathetic and parasympathetic systems was computed. PHF was obtained within a band centered at respiratory rate. Comparing with baseline values, 7 patients showed an increased PHF in post-CR, suggesting an increase of parasympathetic activity.
\end{abstract}

\section{Introduction}

The autonomic nervous system (ANS) plays an important role in the maintenance of systemic homeostasis, exerting a control on essential functions such as cardiac and respiratory functions, thermoregulation and hormonal secretion.

An autonomic dysfunction is frequently observed in patients requiring intensive care unit (ICU) admission [1,2]. Although the incidence of ANS dysfunction in critically ill patients is not exactly established, it is clear that its oc- currence adversely impacts on clinical prognosis, mortality and morbidity [3].

ANS disturbances may produce diverse and unexpected consequences beyond the common deficits associated with the dysfunction of parasympathetic system. For instance, critically ill patients are also at risk of neurocognitive impairments that may persist after hospital discharge. Among various pathophysiological mechanisms proposed to explain neurocognitive impairments in critically ill patients, parasympathetic dysfunction leading cholinergic deficiency seems one of the most viable to explain the development of long-term sequelae. During critical illness a release of inflammatory mediators (e.g., cytokines) can spread to distant organs, including the brain through circumventricular organs and the choroid plexus, which have no blood brain barrier [4]. Increased levels of cytokines and other inflammatory mediators have been associated with neurocognitive impairments [5].

Studies have shown that the parasympathetic nervous system acts as a physiological regulator of the inflammatory response to immune system activation. Recently, a potential role of vagus nerve (i.e. vagal anti-inflammatory pathway) in inflammatory conditions has emerged, and it has been postulated that parasympathetic stimulation via vagus nerve reduces production of systemically active cytokines [6]. Decreased vagus nerve activity is associated with increased morbidity and mortality [7].

A widely used approach to measuring vagus nerve activity in humans is based on cardiac physiology. Heart rate is generated by action potentials controlled by the vagus nerve innervation to the sinoatrial node of the heart, 
where vagus nerve-dependent acetylcholine release essentially prolongs the time to the next heartbeat, thus slowing the pulse. Heart rate variability (HRV) analysis, a non invasive technique that examines beat-to-beat variations in heart rate, has been used successfully to assess vagal activity [8].

It is possible that monitoring vagus nerve activity via HRV may prove to be a measure of the integrity of the ANS, since HRV as a biomarker of cardiac vagal activity could serve to detect physiological deterioration or response to therapy in critically ill patients. Furthermore, prefrontal activation, which has also been related to changes in HRV, could help to strengthen the ANS integrity via the vagus nerve stimulation.

Cognitive rehabilitation (CR) is a behavioral therapy that has proven to be effective improving cognitive deficits in clinical populations with abnormalities in brain activation patterns. In a recent review authors conclude that neural changes associated with CR converge in frontal regions, including cingulate cortex, prefrontal and middle frontal areas [9], the same areas involved in the balance of sympathetic and parasympathetic branches of ANS.

The objective of this work is to explore the effect of the neurocognitive intervention targeted to improve prefrontal activation over ANS measured by HRV in a sample of critically ill patients.

\section{Methods and materials}

\subsection{Database}

17 critically ill patients were included in this study with the following inclusion criteria: patients aged 18 to 85 years, having received mechanical ventilation for at least 24 hours, scoring more than 8 on the Glasgow Coma Scale (GCS) and -1 to +1 on the Richmond Agitation Sedation Scale (RASS) and haemodynamically stable. Patients with previous neurologic pathology or focal brain injury before ICU admission, patients with serious psychiatric pathology or mentally retarded, and patients with sensory impairments were excluded from the study. Also, closest relatives agreed the subject to be included in the study. The clinical study was approved by the human subjects protection committe (CEIC).

From them, 4 patients had a forced termination of the CR due to feeling tired, 7 patients were still mechanically ventiled (MV) during the CR and 2 of them suffered from delirium. Diagnosis includes intestinal perforation, peritonitis, septic shock, politrauma, pneumonia, hemorrhagic shock, toxic intake, pancreatitis, esophageal perforation and acute respiratory failure. Clinical and sociodemographic characteristics of the sample are summarized in Table 1, presented as mean (M) and standard deviation (SD), unless otherwise noted.

\begin{tabular}{|llcc|}
\hline Age (M,SD) & Male & 64.31 & 10.47 \\
Gender (N,\%) & Female & 5 & 70.59 \\
& & 24.31 & 9.53 \\
APACHE-II (M,SD) & 9.5 & 4.53 \\
SOFA (M,SD) & 1.13 & 2.26 \\
RASS (M,SD) & 24.94 & 30.17 \\
Length of ICU stay, & & \\
days (M,SD) & 18.69 & 31.27 \\
$\begin{array}{l}\text { Duration of intubation, } \\
\text { days (M,SD) }\end{array}$ & & \\
$\begin{array}{l}\text { Duration of sedation, } \\
\text { days (M,SD) }\end{array}$ & 7.69 & 8.55 \\
$\begin{array}{l}\text { Septic Shock (N,\%) } \\
\text { Cardiac arrest (N,\%) }\end{array}$ & 9 & 52.94 \\
\hline
\end{tabular}

Table 1. Descriptives. APACHE-II: Acute Physiology and Chronic Health Evaluation II; SOFA: Sequential Organ Failure Assessment; RASS: Richmond Agitation-Sedation Scale.

Patients were monitorized for 20 minutes before, during and after the CR. During the monitorization, the electrocardiogram (ECG) and the respiratory signal were obtained at a sampling rate of $200 \mathrm{~Hz}$.

Neurocognitive intervention: The Early Neurocognitive Rehabilitation in Intensive Care -ENRIC protocol- has been develop in order to apply cognitive stimulation in critically ill patients during ICU stay. The neurocognitive intervention is supported by an interactive platform of advanced signals and image computation, and includes a relaxation and low cognitive load exercises software specifically designed for critically ill patients. One of the main objectives of the module is to offer intuitive interaction, as well as a logical and natural response. Kinect, a Microsoft gesture recognizing device, capable of tracking an image of the distance between the user and the camera in real time, thus capturing user movements and gestures, is used. The stimulation module shows a pleasant and relaxing virtual environment where the patient can walk through. During the session time a virtual avatar goes along with the patient, orienting, motivating and inviting him/her to relax. In order to get this objective, different active and passive exercises through the virtual environment have been developed:

Passive exercises: The objective of these exercises is focused in relaxation of the patient while simple attentional functions are stimulated. These passive exercises have been especially designed for patients with difficulties of mobility and alertness, due to their low interaction level.

Active exercises: The aim of the active exercises is focused in the cognitive stimulation of complex functions (i.e. complex attentional functions and executive functions), and therefore, these exercises involve high level of cognitive requirement. 


\subsection{Data analysis}

Spectral analysis is made in consecutive 2 minute segments with an overlap of 1 minute. The classic periodogram is applied to the HRV and respiratory signal to obtain their power spectral densities (PSD).

Respiratory signal: The respiratory frequency $f_{r}$ is set as the frequency at which the PSD of the respiratory signal is maximum within the band $[0.15,0.8] \mathrm{Hz}$. Then, 2 eligible bands for high frequency (HF) are defined, always centered around $f_{r}$ and within the band between $0.15 \mathrm{~Hz}$ and half the mean heart rate. These 2 bands are: $\Omega_{\mathrm{HF}}=\left[f_{r^{-}}\right.$ $\left.0.075, f_{r}+0.075\right] \mathrm{Hz}$ and $\Omega_{\mathrm{HFe}}=\left[f_{r}-0.15, f_{r}+0.15\right]$ Hz. Subsequently, the percentage of power in $\Omega_{\mathrm{HF}}$ with respect to the total power in $\Omega_{\mathrm{HFe}}$ is computed. If this percentage is above 0.7 , the spectrum around $f_{r}$ is considered to be "peaky" enough and $\Omega_{\mathrm{HF}}$ is used as HF band, otherwise the spectrum is more spread out and $\Omega_{\mathrm{HFe}}$ is used to cover it.

HRV signal: A 4-Hz-sampled instantaneous heart rate signal $d_{\mathrm{HR}}(n)$ is derived from the ECG. The very low frequency component is obtained by low-pass filtering $d_{\mathrm{HR}}(n)$ using a cut-off frequency of $0.03 \mathrm{~Hz}$ and denoted $d_{\mathrm{HRM}}(n)$. The HRV signal is obtained as $d_{\mathrm{HRV}}(n)=d_{\mathrm{HR}}(n)-d_{\mathrm{HRM}}(n)$. According to the integral pulse frequency modulation model, the modulating signal carrying information on the ANS, $m(n)$, is obtained as $m(n)=d_{\mathrm{HRV}}(n) / d_{\mathrm{HRM}}(n)$ [10].

From the PSD of $m(n)$, the power in the low (LF) and high spectral bands are obtained: $P_{\mathrm{LF}}$ for the LF band $(0.04-0.15 \mathrm{~Hz})$ and $P_{\mathrm{HF}}$ for the $\mathrm{HF}$ band (centered at $\left.f_{r}\right)$. In addition, the total power in both LF and HF bands $\left(P_{\text {TOT }}\right)$, and the normalized power in LF band with respect to $P_{\text {Tor }}$ $\left(P_{\mathrm{LFn}}\right)$ are also computed.

For the analysis, the changes in the power during the session (SES) with respect to the basal state (PRE) is obtained as $\Delta P_{\mathrm{S}}^{\mathrm{SES}}=\left(P_{\mathrm{S}}^{\mathrm{SES}}-P_{\mathrm{S}}^{\mathrm{PRE}}\right) / P_{\mathrm{S}}^{\mathrm{PRE}}$, with $P^{\mathrm{PRE}}$ and $P^{\mathrm{SES}}$ meaning the power parameter before and during the CR, and $\mathrm{S}=\{\mathrm{LF}, \mathrm{HF}, \mathrm{LFn}, \mathrm{TOT}\}$. For changes after the session, the analysis are similar: $\Delta P_{\mathrm{S}}^{\mathrm{POS}}=\left(P_{\mathrm{S}}^{\mathrm{POS}}-P_{\mathrm{S}}^{\mathrm{PRE}}\right) / P_{\mathrm{S}}^{\mathrm{PRE}}$, with $P^{\mathrm{POS}}$ meaning the power parameter after the $\mathrm{CR}$.

\section{Results}

Results are presented for 3 different groups of patients: patients who did not suffer from delirium and were not MV (G1, 11 patients), patitents who were MV (G2, 4 patients), and patients who suffered from delirium (G3, 2 patients).

G1: During the session, 4 out of 11 patients present an increase in $\Delta P_{\mathrm{HF}}^{\mathrm{SES}}$ and $\Delta P_{\mathrm{TOT}}^{\mathrm{SES}}$ and a decrease in $\Delta P_{\mathrm{LFn}}^{\mathrm{SES}}, 4$ present the opposite response, and 3 present a decrease in the three parameters. 6 out of 11 patients show an increase in $\Delta P_{\mathrm{HF}}^{\mathrm{pos}}$ after the session and 4 of them also present a decrease in $\Delta P_{\mathrm{LFn}}^{\mathrm{POS}}$ and an increase in $\Delta P_{\mathrm{TOT}}^{\mathrm{POS}}$.

G2: 2 out of 4 patients had a forced termination of the cognitive rehabilitation due to feeling tired. The 2 patients who underwent the whole session present a decrease in all parameters during the intervention. After the session, those patients with a forced termination present a decrease in all parameters. The other two patients present opposite changes: one of them has an increase in $\Delta P_{\mathrm{HF}}^{\mathrm{pOs}}$ and a decrease in $\Delta P_{\mathrm{LF}}^{\mathrm{POS}}$, while the other has a decrease in $\Delta P_{\mathrm{HF}}^{\mathrm{POS}}$ and an increase in $\Delta P_{\mathrm{LFn}}^{\mathrm{POs}}$; however both present an increase in $\Delta P_{\mathrm{TOT}}^{\mathrm{POS}}$.

G3: There are 2 patients with delirium and both were also MV. Also, one of them had a forced termination of the session. During the session, both patients present an increase in $\Delta P_{\mathrm{HF}}^{\mathrm{SES}}$ and $\Delta P_{\mathrm{TOT}}^{\mathrm{SES}}$ and a decrease in $\Delta P_{\mathrm{LFn}}^{\mathrm{SES}}$. After the session, both patients present an increase in $\Delta P_{\mathrm{LFn}}^{\mathrm{POS}}$ and a decrease in $\Delta P_{\mathrm{HF}}^{\mathrm{POS}}$ while $\Delta P_{\mathrm{TOT}}^{\mathrm{POS}}$ decreases.

\section{Discussion}

HRV parameters are widely used to assess the ANS activity. In this work, power in the HF band is used as an index of the parasympathetic activity, while the sympathetic activity is related to the normalized power in the LF band. Also, the total power is used as a measure of the variance of the HRV. Several researchers have demonstrated that low or decreasing HRV in intensive care unit (ICU) patients reflects greater severity of illness and predicts subsequent deterioration and mortality [11]. During mental and physical tasks, an increase in sympathetic activity has been observed [12]. Therefore, our hypothesis is that an activation of the sympathetic activity should be observed during the neurocognitive session due to the mental and physical effort. After the session, when cognitive and physical demands disappear, vagal system takes over and we expected that the parasympathetic activity should be increased.

Results during the session do not seem to follow any pattern. A possible explanation could be the medicines that patients were taking, which greatly alters ANS activity. Results after the session are more promising, since there are 6 patients with a higher $P_{\mathrm{HF}}$ associated with a higher parasympathetic activity, and 4 of them also present a reduced sympathetic activity. This behavior dominates in the "moderately ill" patients, i.e., those who were not MV, did not suffer from delirium and did not have an abrupt termination of the neurocognitive intervention. This suggests that this therapy could improve the ANS balance in these patients, while other patients with worse conditions could need more sessions or even a different kind of intervention.

Some MV patients present a very irregular breathing signal. They do not adapt to the reference frequency and they are constantly fighting against the machine. These patients had also a forced termination of the session, and present a decrease in all parameters. However it is not clear if this decrease in the HRV power is related to the irregular breathing or to the medication, there are very few MV 
patients in this database to extract any conclusion.

It remains unclear if delirium can be caused by an autonomic dysfunction. The only study published to date found no differences comparing patients with and without delirium in HF and low to high frequency ratio [13]. In our sample, patients suffering from delirium in the moment of the CR session showed an inverse pattern as we expected. It is possible that patients with delirium showed severe impairs in their attentional capacities that did not allow to follow the CR session, so intervention had no effect over ANS in these patients. Further investigations are needed to elucidate if neurocognitive therapies might have any effect on cognition in patients suffering from delirium states. It is important to consider that delirium patients in our sample were also MV, so interpretation of these results should be taken carefully.

Critically ill patients in medical and surgical ICUs are a highly heterogeneous group of patients (including a wide range age, ICU admission diagnosis and comorbidities). Therefore, HRV in critically ill patients may be affected by various factors (sedation, ventilator pattern, effect of drugs administered); thus, the heterogeneity of the sample patients and the different clinical practices used are important limitations of this study. The extension of this work is to analyze subsequent sessions for each patient, and correlate the effect of the neurocognitive intervention on the ANS with the severity of the disease or other factors such as the effect of drugs they are taking. Also, further studies are needed involving a large number of critically ill patients.

\section{Conclusion}

This study analyses the autonomic nervous system activity by analysing the spectral parameters of the heart rate variability in critically ill patients who undergo cognitive rehabilitation. Comparing with baseline values, 7 patients showed an increase in the HF power after the session, suggesting an increase of parasympathetic activity, while 5 of them presented a decrease in the normalized LF power, suggesting a decrease of the sympathetic activity. However, critically ill patients are very diverse, already have altered the ANS and they take medicines which also modify its behaviour.

\section{Acknowledgements}

This work has been supported by the Diputación General de Aragón (DGA), Spain, through a fellowship with reference B195/12, by MINECO and FEDER (EU), under project TIN2014-53567-R, by Grupo Consolidado BSICoS ref:T96 from DGA, by Aragón Institute of Engineering Research (I3A), IIS Aragón, by University of Zaragoza UZ2014TEC-01, European Social Fund (EU) and by a collaborative project from CIBERES, CIBER-BBN and SEPAR. The computation was performed by the ICTS NANBIOSIS, more specifically by the High Performance Computing Unit of the CIBER-BBN at the University of Zaragoza. This work has also been financially supported by Fundació La Marató TV3
181/U/2011, ISCIII PI13/02204, ES15PINT007 and Fundació Parc Taulí. No funding organization or sponsor was involved in the preparation, review, or approval of the manuscript.

\section{References}

[1] Schmidt H, Hoyer D, et al. The alteration of autonomic function in multiple organ dysfunction syndrome. Crit Care Clin 2008;24(1):149-163, ix.

[2] Wieske L, Chan Pin Yin DR, et al. Autonomic dysfunction in ICU-acquired weakness: a prospective observational pilot study. Intensive Care Med 2013;39(9):1610-1617.

[3] Ryan ML, Ogilvie MP, Pereira BM, et al. Heart rate variability is an independent predictor of morbidity and mortality in hemodynamically stable trauma patients. J Trauma 2011;70(6):1371-1380.

[4] López-Aguilar J, Quilez ME, Martí-Sistac O, GarcíaMartín C, Fuster G, Puig F, et al. Early physiological and biological features in three animal models of induced acute lung injury. Intensive Care Med 2010;36:347-355.

[5] Reichenberg A, Yirmiya R, Schuld A, Kraus T, Haack M, Morag A, Pollmacher T. Cytokine-associated emotional and cognitive disturbances in humans. Arch Gen Psychiatry 2001;58(5):445-452.

[6] Zitnik RJ. Treatment of chronic inflammatory diseases with implantable medical devices. Cleve Clin J Med 2011;78 Suppl 1:S30-34.

[7] Huston JM, Tracey JC. The pulse of inflammation: heart rate variability, the cholinergic anti-inflammatory pathway and implications for therapy. Journal of Internal Medicine 2010;269:7.

[8] Thayer JF, Yamamoto SS, et al. The relationship of autonomic imbalance, heart rate variability and cardiovascular disease risk factors. Int J Cardiol 2010;141(2):122-131.

[9] Thorsen AL, Johansson K, et al. Neurobiology of cognitive remediation therapy for schizophrenia: a systematic review. Front Psychiatry 2014;5:103.

[10] Bailón R, Laouini G, Grao C, Orini M, Laguna P, Meste O. The integral pulse frequency modulation with time-varying threshold: application to heart rate variability analysis during exercise stress testing. IEEE Trans Biomed Eng 2011; 58 (3):642-652.

[11] Piepoli M, Garrard CS, Kontoyannis DA, Bernardi L. Autonomic control of the heart and peripheral vessels in human septic shock. Intensive Care Med 1995;21:112.

[12] Garde AH, Laursen B, Jørgensen AH, Jensen BR. Effects of mental and physical demands on heart rate variability during computer work. Eur J Appl Physiol 2002;87(4-5):456461.

[13] Zaal IJ, van der Kooi AW, van Schelven LJ, Oey PL, Slooter AJ. Heart rate variability in intensive care unit patients with delirium. J Neuropsychiatry Clin Neurosci 2015; 27(2):e112-116.

Address for correspondence:

David Hernando (dhernand@unizar.es). Mariano Esquillor s/n, Ed. I+D+i, L4.0.04, 50018, Zaragoza (Spain). 